\title{
Gaia 17bpi: An FU Ori-type Outburst
}

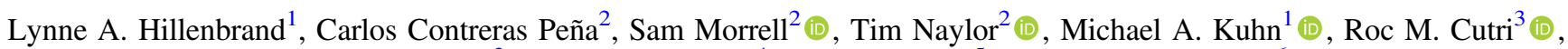 \\ Luisa M. Rebull ${ }^{3}$ (D), Simon Hodgkin ${ }^{4}$, Dirk Froebrich ${ }^{5}$, and Amy K. Mainzer ${ }^{6}$ \\ ${ }^{1}$ Department of Astronomy, California Institute of Technology, Pasadena, CA 91125, USA \\ ${ }^{2}$ School of Physics, University of Exeter, Stocker Road, Exeter, Devon EX4 4QL, UK \\ ${ }^{3}$ IPAC, California Institute of Technology, Pasadena, CA 91125, USA \\ ${ }^{4}$ Institute of Astronomy, University of Cambridge, Madingley Road, Cambridge CB3 0HA, UK \\ ${ }^{5}$ Centre for Astrophysics \& Planetary Science, The University of Kent, Canterbury, Kent CT2 7NH, UK \\ ${ }^{6}$ JPL, 4800 Oak Grove Drive, Pasadena, CA 91109, USA \\ Received 2018 October 6; revised 2018 November 9; accepted 2018 November 15; published 2018 December 20
}

\begin{abstract}
We report on the source Gaia 17bpi and identify it as a new, ongoing FU Ori-type outburst, associated with a young stellar object. The optical light curve from Gaia exhibited a 3.5 mag rise with the source appearing to plateau in mid-/late 2018. Mid-infrared observations from NEOWISE also show a $>3$ mag rise that occurred in two stages, with the second one coincident with the optical brightening, and the first one preceding the optical brightening by $\sim 1.5 \mathrm{yr}$. We model the outburst as having started between October and December of 2014. This wavelength-dependent aspect of young star accretion-driven outbursts has never been documented before. Both the mid-infrared and the optical colors of the object become bluer as the outburst proceeds. Optical spectroscopic characteristics in the outburst phase include: a GK-type absorption spectrum, strong wind/outflow in, e.g., Mgb, $\mathrm{NaD}, \mathrm{H} \alpha, \mathrm{K}$ I, O I, and Ca II profiles, and detection of $\mathrm{Li}$ I $6707 \AA$. The infrared spectrum in the outburst phase is similar to that of an M-type spectrum, notably exhibiting prominent $\mathrm{H}_{2} \mathrm{O}$ and ${ }^{12} \mathrm{CO}(2-0)$ bandhead absorption in the $K$ band, and likely He I wind in the $Y$ band. The new FU Ori source Gaia 17bpi is associated with a littlestudied dark cloud in the galactic plane, located at a distance of $1.27 \mathrm{kpc}$.
\end{abstract}

Key words: circumstellar matter - stars: activity - stars: general - stars: pre-main sequence - stars: variables: general - stars: winds, outflows

Supporting material: data behind figures

\section{Introduction}

The accretion paradigm for young stars is reviewed by Hartmann et al. (2016). It features an early spherical-like infall from a hydrostatic core of slowly rotating molecular and dusty material, the buildup of (proto)stellar mass through a combination of the direct collapse and accretion from a disk (mediated by a fraction of the mass that is lost in accretiondriven winds/outflows), and finally, a late-accretion phase that competes with planet formation and remnant winds in completely depleting the disk. The initially high accretion rates are believed to generally decline with age. However, our physical understanding of star formation and stellar mass assembly currently relies on episodic accretion, or punctuated periods of enhanced mass accretion/outflow, in order to build up the needed stellar mass on the required timescales.

Based on a small sample of "classical" FU Ori stars (Herbig 1977) and a few subsequently discovered FU Ori-like objects-those whose transition to the outburst state had not been observed-Hartmann \& Kenyon (1996) summarized the basic scenario of episodic accretion. They envisioned that enhanced accretion would occur more frequently in more massive disks at early protostellar stages, and thus that the rate of FU Ori events would be higher for protostars and lower for optically revealed pre-main-sequence stars. Subsequent detailed modeling of instabilities arising in the inner disk (Bae et al. 2014) or the outer disk (Vorobyov \& Basu 2015) has produced quantitative predictions concerning the amplitudes, durations, and duty cycles of episodic accretion in young stellar objects (YSOs).
A long-standing problem for the importance placed on the FU Ori scenario in building up stellar mass is that FU Ori outbursts are rare. Over the past seven decades, fewer than 13 actual FU Ori outbursts have been recorded, with another $\sim 13$ sources identified as FU Ori-like based on their present spectra and spectral energy distributions (SEDs) obtained in a hypothetical post-outburst state (Reipurth \& Aspin 2010; Connelley \& Reipurth 2018). Although the discoveries are increasing in number with time (Figure 73 in Reipurth 2016), the rate of FU Ori outbursts remains rather poorly constrained empirically. This is an especially notable gap when compared to our state of knowledge regarding event rates for, e.g., cataclysmic variable and other novae, supernovae of various classes, and even tidal disruption events.

Modern all-sky and all-hemisphere time domain surveys have the potential to better constrain the true FU Ori rate (Hillenbrand \& Findeisen 2015) and better illuminate the diversity of young star outbursts of various types, e.g., FU Orilike versus V1647 Ori-like versus EX Lup-like (Contreras Peña et al. 2017a, 2017b). Only when the full phase space of YSO variability is more completely mapped out can we improve our understanding of how stars gain their mass.

The Gaia mission is one such photometric survey, performing repeated scans of the sky primarily directed toward establishing accurate and precise astrometry. However, changes in source brightness trigger alerts that are made publicly available. ${ }^{7}$ These pages are monitored by groups wishing to identify objects of interest to their science goals and perform

\footnotetext{
Gaia Alerts; http://gsaweb.ast.cam.ac.uk/alerts.
} 


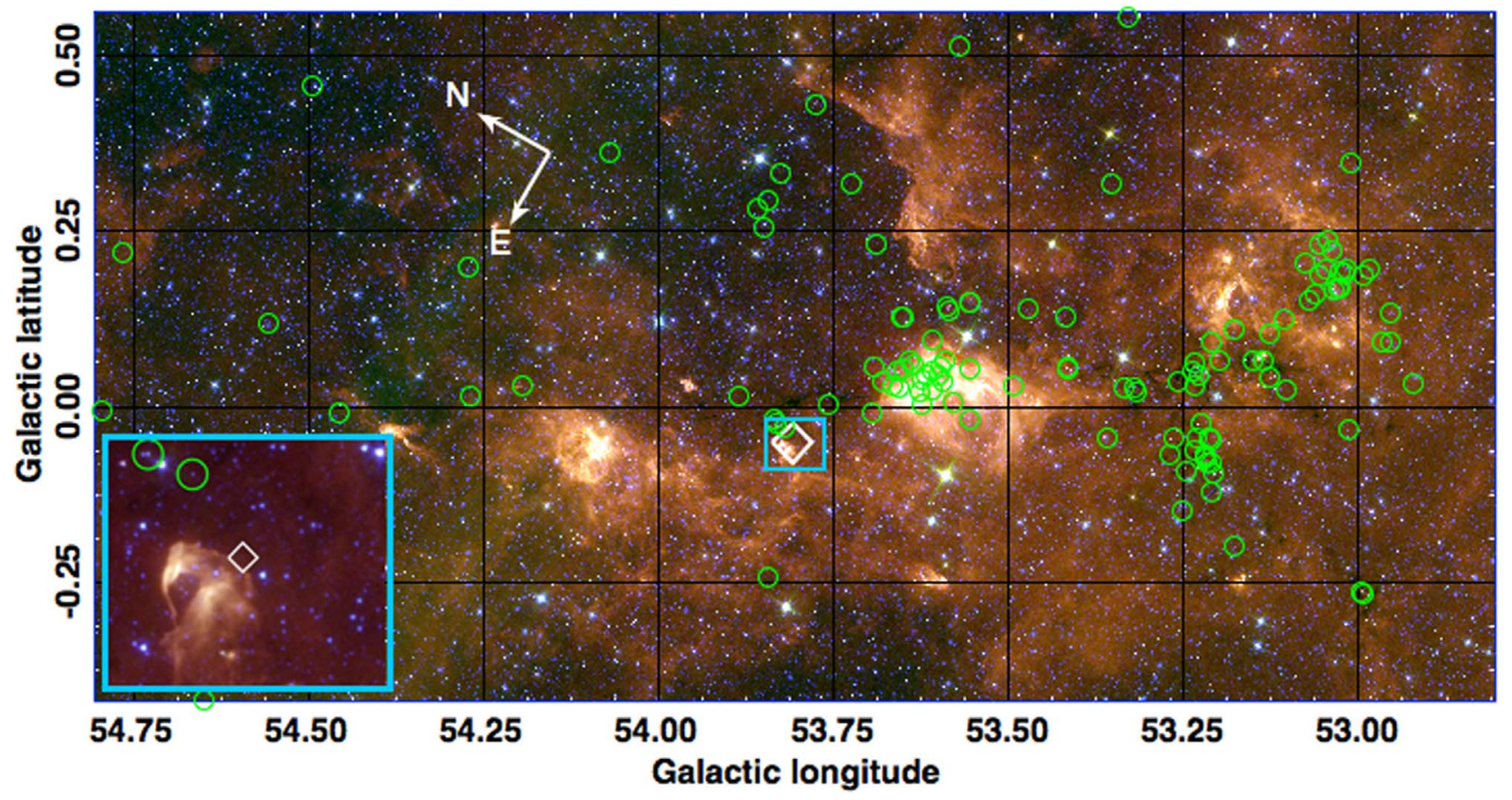

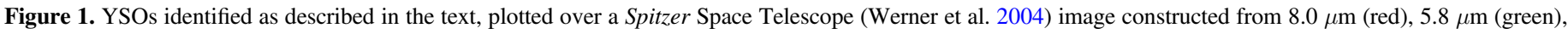

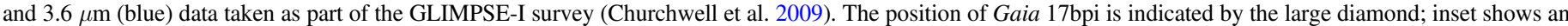
expanded view $4.5 \times 4^{\prime}$ in size illustrating the adjacent small $\mathrm{H}$ II region HRDS G053.822-00.057.

follow-up observations. An alert for Gaia 17bpi was issued on 2017 June 21, following a 2 mag brightness increase relative to observations from the earliest Gaia epochs. The source has continued to brighten.

While the light curve of Gaia 17bpi exhibits an FU Ori-like rise, several classes of large-amplitude pulsating variables have similarly slow rise times, as can active galactic nuclei. However, the location of Gaia 17bpi in proximity to an optically opaque cloud and region of infrared nebulosity suggested that further investigation of the source was warranted.

In this paper, we report on the environment of the new outburst source Gaia 17bpi (Section 2), on the characteristics of the pre-outburst object (Section 3), and on the substantial rise relative to previous photometry exhibited in the Gaia light curve as well as in NEOWISE observations, including followup optical photometry (Sections 4.1-4.3). In Section 4.4, we present new outburst-era spectroscopy at optical and infrared wavelengths, and Section 5 contains a short discussion and our conclusions.

\section{The Star-forming Region Containing the New Outburst Source}

Gaia $17 \mathrm{bpi}$ is associated with a previously faint optical $(r<22 \mathrm{mag}$ ), near-infrared, and mid-infrared point source. The position (see Figure 1) is located toward the northern end of the elongated G53.2 "infrared dark cloud," just outside of a particularly opaque region on optical and infrared images, and adjacent to a small H II region cataloged as HRDS G053.82200.057 (Anderson et al. 2014). The modest star-forming region is hardly studied.

The only previous relevant publication covering objects in the vicinity of Gaia $17 \mathrm{bpi}$ is by Kim et al. (2015). These authors illustrate the morphology of the dust (based on
Bolocam) and gas (based on GRS) relative to the mid-infrared emission and absorption (see their Figure 1). Gaia 17bpi appears to be on the periphery of the cloud, beyond the $\mathrm{CO}$ gas contours that also delineate the optically opaque region, but between two millimeter-wavelength dust clumps. Kim et al. (2015) used Spitzer photometry to identify several hundred young stars as likely members of the star-forming region that extends in a narrow filament over several degrees to the southeast of Gaia 17bpi, though not including the newly outbursting source that we report here. Kim et al. (2015) quote a kinematic distance to the cloud of $1.7 \mathrm{kpc}$.

In order to refine the distance and to assess the overall environment of the outburst source, we repeated the Kim et al. (2015) analysis by using the Spitzer/GLIMPSE (Churchwell et al. 2009), 2MASS (Cutri et al. 2003; Skrutskie et al. 2006), and Gaia DR2 (Gaia Collaboration et al. 2018) catalogs. The color criteria of Gutermuth et al. (2009) were used to identify infrared excess sources that are likely YSOs, then Gaia proper motions and parallaxes were used to down-select to a sample of reliable members. From infrared excess alone, there is substantial contamination from dusty giants in the background. From Gaia alone, it is difficult to identify the association due to an insufficient population of stars that would stand out in proper-motion/parallax space. As in Kim et al. (2015), Gaia $17 \mathrm{bpi}$ was not selected by our mid-infrared selection methods, mainly because in the available Spitzer catalog data it is detected in only the two shortest (3.6 and $4.5 \mu \mathrm{m}$ ) photometry bands. The spatial distribution of our infrared excess and kinematically selected sources exhibits two clumps to the southeast of Gaia 17bpi, coincident with the morphology of the opacity in optical/infrared images. Again, Gaia $17 \mathrm{bpi}$ is found to be located on the periphery of the star-forming region.

We assess the cloud distance based on examination of the Gaia parallax distribution of the infrared excess sources, using a weighted median approach identical to that described in 
Table 1

Newly Derived Infrared Photometry ${ }^{\mathrm{a}}$ for the Gaia 17bpi Progenitor

\begin{tabular}{lccc}
\hline \hline Instrument & Wavelength $(\mu \mathrm{m})$ & Flux (Jy) & Error (Jy) \\
\hline Spitzer/IRAC & 3.6 & $312.26 \mathrm{e}-6$ & $43.09 \mathrm{e}-6$ \\
Spitzer/IRAC & 4.5 & $293.32 \mathrm{e}-6$ & $43.34 \mathrm{e}-6$ \\
Spitzer/IRAC & 5.8 & $499.99 \mathrm{e}-6$ & $142.61 \mathrm{e}-6$ \\
Spitzer/IRAC & 8.0 & $754.89 \mathrm{e}-6$ & $247.29 \mathrm{e}-6$ \\
Spitzer/MIPS & 24 & $<0.058$ & $\ldots$ \\
Herschel/PACS & 70 & $<1.14$ & $\ldots$ \\
Herschel/PACS & 160 & $<3.92$ & $\ldots$ \\
Herschel/SPIRE & 250 & $<3.24$ & $\ldots$ \\
Herschel/SPIRE & 350 & $<2.61$ & $\ldots$ \\
Herschel/SPIRE & 500 & $<2.76$ & $\ldots$ \\
\hline
\end{tabular}

Notes.

${ }^{a}$ Observations were taken in 2004 as part of the Spitzer GLIMPSE and MIPSGAL programs.

Kuhn et al. (2019). Our value of $1270_{-70}^{+80} \mathrm{pc}$ (including both random and systematic error) is approximately $25 \%$ closer than the previous kinematic distance estimate. The total cloud mass reported by $\mathrm{Kim}$ et al. (2015), correcting for the distance revision, becomes a few $\times 10^{4} M_{\odot}$. The cloud is comparable in its filamentary morphology and its size and mass to the Taurus dust cloud and molecular gas complex (Pineda et al. 2010). The cloud radial velocity reported by Kim et al. (2015) is $22.9 \pm 1.0 \mathrm{~km} \mathrm{~s}^{-1}$, which if an LSR value corresponds to $v_{\text {helio }}=4.5 \mathrm{~km} \mathrm{~s}^{-1}$.

\section{The Pre-outburst Object}

\subsection{SED Data Collection}

The SED of the pre-outburst object was assembled from catalog data originating from the Gaia DR2, PanSTARRS DR1, IPHaS, 2MASS, and Spitzer/GLIMPSE-I surveys, as well as new data reduction of Spitzer and Herschel images. The results of the reductions described below are given in Table 1.

For the Spitzer data, the GLIMPSE catalog contains measurements only at the shortest wavelengths, 3.6 and $4.5 \mu \mathrm{m}$. In order to better characterize the infrared SED, we downloaded from IPAC/IRSA all Spitzer image data from $\operatorname{IRAC}(3.6,4.5,5.8,8 \mu \mathrm{m})$ and $\operatorname{MIPS}(24,70 \mu \mathrm{m})$, and all Herschel data from PACS $(70,160 \mu \mathrm{m})$ and SPIRE $(250,350$, $500 \mu \mathrm{m})$. The source is apparent in all four IRAC bands, but at the longer wavelengths there is contamination from a very bright source to the northeast and only upper limits could be derived, at much higher levels than typical in less confused regions.

The position was observed at mid-infrared wavelengths as part of the GLIMPSE program (Benjamin et al. 2003; Churchwell et al. 2009) with IRAC in late 2004. The mosaics we used were assembled as part of the Spitzer Enhanced Imaging Products ${ }^{8}$ that summed up all available data from the cryogenic phase of the mission. GLIMPSE is a relatively shallow survey, but the target is clearly visible in the IRAC-1 $(3.6 \mu \mathrm{m})$ and IRAC-2 $(4.5 \mu \mathrm{m})$ bands; it is faint but present in IRAC-3 $(5.8 \mu \mathrm{m})$ and IRAC-4 $(8 \mu \mathrm{m})$. Aperture photometry

\footnotetext{
8 SEIP; https://irsa.ipac.caltech.edu/data/SPITZER/Enhanced/SEIP/overview. html.
}

was performed at the location, using an aperture of 3 native pix, with an annulus of 3-7 native pix. (A native pixel is 1 !'2.) Aperture corrections of $1.124,1.127,1.143$, and 1.234 were used, as described in the IRAC Instrument Handbook. ${ }^{9}$ Reported errors are statistical and do not include systematic effects.

This region was also observed by MIPS at $24 \mu \mathrm{m}$ as part of the MIPSGAL program (Carey et al. 2009) in late 2005, and mosaics regenerated as part of SEIP. However, the source was not detected. Calculating photometric limits at this location is complicated by the nearby bright ISM; we performed aperture photometry at the location as if there was a source there, following suggestions from the MIPS Instrument Handbook, ${ }^{10}$ that is, 5! 6 aperture, annulus 5!"6-10". 4 , and an aperture correction of 2.05 .

This region was also observed by Herschel (Pilbratt et al. 2010) in late 2011 as part of the Hi-GAL program (Molinari et al. 2010) using PACS and SPIRE. The source is not visible in the Herschel High-Level Images, ${ }^{11}$ which sum up all available data. As with MIPS-24, limits are not terribly constraining because of the bright ISM near the region. Aperture photometry obtained from the corresponding instrument handbook was performed at the location to estimate upper limits in each available band (PACS: 70 and $160 \mu \mathrm{m}$; SPIRE: $250,350$, and $500 \mu \mathrm{m})$.

\subsection{SED Analysis}

The $0.5-4.5 \mu \mathrm{m}$ photometric measurements and the longerwavelength upper limits are shown in Figure 2. It may be important to note that the pre-outburst photometry consists of measurements taken at different times, with the optical grizy data taken in 2012, the near-infrared $J H K$ data taken in 1999, and the IRAC1,2,3,4 measurements taken in 2004. As these sets are noncontemporaneous, the compiled SED could be deceptive.

Photospheric models ranging in temperature from 2900-3200-3500-4000-4500 K from NextGen2 (following Hauschildt et al. 1999) result in acceptable fits to the Wien part of the SED for corresponding visual extinction values of 1-2-3-4-5 mag, respectively. Photospheres warmer than $\sim 5500 \mathrm{~K}$ cannot be reddened enough to match the bluer wavelengths without exceeding the measurements around and beyond the SED peak. Photospheres cooler than $\sim 2700 \mathrm{~K}$ do not match at the bluer wavelengths, even unreddened.

Hotter temperatures minimize the near-infrared excess; however, these warmer temperatures do not match the $r$-band to $G$-band to $i$-band data as well as cooler temperatures do. Temperatures cooler than $\sim 4000 \mathrm{~K}$ imply a near-infrared excess, the amplitude of which increases as the assumed photospheric temperature decreases. The 5.8 and $8.0 \mu \mathrm{m}$ points clearly exceed the expectations from a reddened photosphere of any temperature. Our preferred model, derived by considering a number of different normalization schemes, is the $3500 \mathrm{~K}$ photosphere with $A_{V}=3 \mathrm{mag}$.

\footnotetext{
SSC; http://irsa.ipac.caltech.edu/data/SPITZER/docs/irac/iracinstrumen thandbook/.

${ }^{10} \mathrm{SSC}$; http://irsa.ipac.caltech.edu/data/SPITZER/docs/mips/mipsinstrument handbook/.

${ }^{11}$ HHLI; http://irsa.ipac.caltech.edu/data/Herschel/HHLI/overview.html.
} 


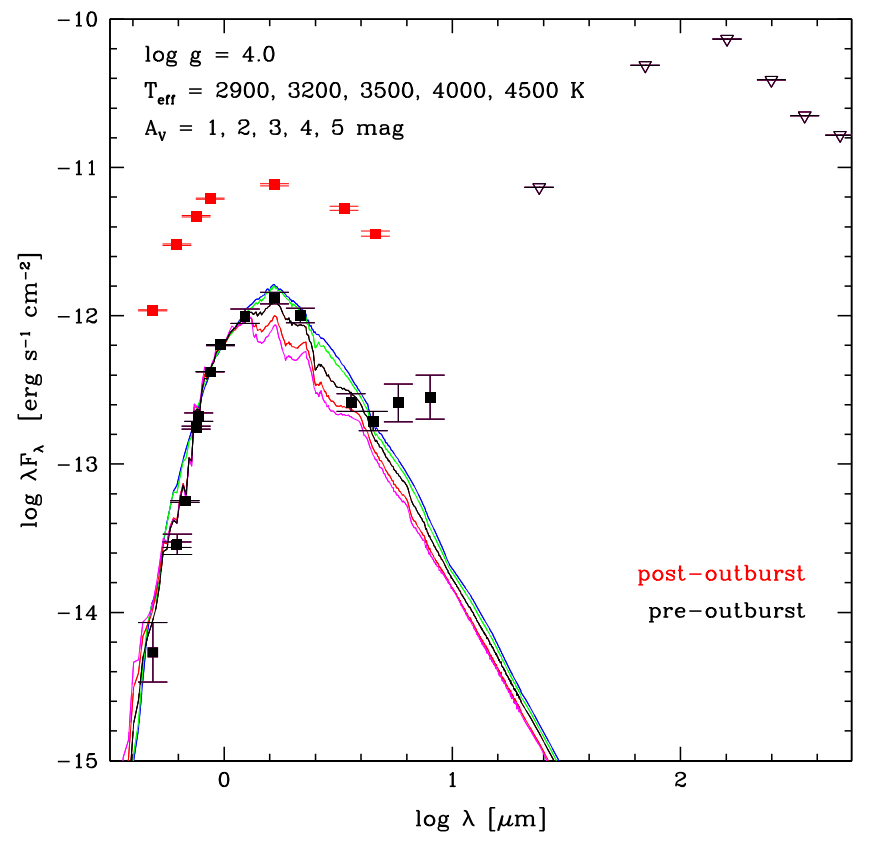

Figure 2. Spectral energy distribution of Gaia 17bpi. Red points are the postoutburst color data presented here. Black points are the assembled pre-outburst photometry with black downward triangles indicating upper limits. Also shown are reddened model photospheres normalized at $z$ band to the pre-outburst photometry. See the text for sources of data and models. The empirical SED in the pre-outburst state is consistent with a photospheric temperature in the range of 2900-4500 K, with more reddening required for the hotter temperatures, and an infrared excess. The excess is clearly implied by the 8.0 and $5.8 \mu \mathrm{m}$ measurements, but at $4.5 \mu \mathrm{m}$ and shorter wavelengths it is dependent on the true photospheric temperature. Note that the pre-outburst sets of grizy, JHK, and IRAC1,2,3,4 measurements were not obtained contemporaneously, and thus the compiled SED could be deceptive.

\subsection{Other Attributes}

Unfortunately, we know little else about the pre-outburst object beyond its location and its colors. It was too faint for parallax or proper motion reporting in Gaia DR2, for example.

Integrating the pre-outburst photometry described above between 0.45 and $4.5 \mu \mathrm{m}$, and assuming the $1.27 \mathrm{kpc}$ distance derived above, a source luminosity of $0.3 L_{\odot}$ is derived for the pre-outburst object. The true value will be slightly higher once the proper reddening correction is determined. This luminosity is seemingly appropriate for a pre-main-sequence star in the above temperature range. The corresponding spectral type would be mid-K to $\mathrm{M}$.

In the Gaia photometry presented below, there is some indication of photometric variability in the earliest $G$-band measurements, taken pre-outburst. Gaia $17 \mathrm{bpi}$ is quoted in the Gaia DR2 catalog at $G=20.44 \pm 0.015$ mag. Given that 13 transits contribute to that measurement, the individual measurements are implied to have an uncertainty of $0.05 \mathrm{mag}$. However, as the uncertainty is based on the scatter between data points, any intrinsic variability will be included in the uncertainty value. Indeed, most sources at this magnitude in the general area have an uncertainty of only $0.005 \mathrm{mag}$. The possible pre-outburst variability of Gaia 17bpi has an amplitude of a few tenths of a magnitude and occurs on a timescale of a few days (Figure 3), consistent with "typical" T Tauri star variability (e.g., Cody \& Hillenbrand 2018; Rebull et al. 2018).

The iPHaS catalog (Barentsen et al. 2014) provides $r-\mathrm{H} \alpha$ color of $0.88 \pm 0.29 \mathrm{mag}$, but the very red $r-i$ color of
$2.63 \pm 0.18 \mathrm{mag}$ (Figure 4) does not make the source an obvious $\mathrm{H} \alpha$ emitter, according to the models in Figure 2 of Barentsen et al. (2013).

The field of Gaia 17bpi was also covered as part of the UWISH2 survey (Froebrich et al. 2011). Consistent with results presented below, the pre-outburst source is detected in continuum $J$-band and $K$-band images (taken 2006 July 11 for the UKIDSS Galactic Plane Survey), but does not show an excess in an $\mathrm{H}_{2}-K$ difference image $\left(\mathrm{H}_{2}\right.$ data taken 2010 November 26). There is thus no signature of a preexisting large-scale shocked outflow from the Gaia 17bpi progenitor, only some jets in the dark filament to the northwest of the source that are not related, and some nebulosity to the northeast that is coincident with the mid-infrared nebulosity illustrated below in Figure 5. If an outflow appears over the next few years, it would be direct evidence for FU Ori outburst triggering of mass ejection.

\section{The Outburst of Gaia 17bpi}

\subsection{Discovery}

At position 19:31:5.590 + 18:27:52.27 (J2000), Gaia 17bpi was flagged by an automated alerts system (Hodgkin et al. 2013; S. T. Hodgkin et al. 2019, in preparation) and announced on the Gaia Alerts public feed ${ }^{12}$ on 2017 June 23. The alert was triggered as a "delta-magnitude detection" based on the source brightening relative to earlier Gaia photometry by $>1$ mag in two consecutive transits of the satellite over the source position (in March and then June, in this case). Among the stream of Gaia Alerts, this particular source was noted as being a potential young star outburst via an ongoing program to identify YSOs that are published on the Gaia Alerts feed. C. Contreras Peña et al. (2018, in preparation) present a full description of this program.

Although Gaia 17bpi was not successfully cross-matched with any specific object in the search catalogs, it was identified using what we term a "vicinity match," that is, as being located within 2 arcmin of a confirmed or candidate YSO. This methodology leverages the fact that YSOs are located in spatially coherent regions of star formation, hence clustered on the sky. Further, it acknowledges that the current census information for many nearby star-forming regions remains incomplete and uses photometric variability to flag potential YSOs that are previously uncataloged. In fact, Gaia $17 \mathrm{bpi}$ has five stars falling in the vicinity of the alert coordinates, given in Table 2 .

\subsection{Assembly of Light-curve Data \\ 4.2.1. Optical}

Photometry from the Gaia mission was downloaded from the alerts service webpage, with the last update for our analysis occurring on 2018 September 10. Only Gaia G-band measurements are available, and error estimates are not included. We do not reproduce the numbers here, as the photometric quality should continue to improve as the mission proceeds.

The recent Gaia light curve was supplemented by heritage catalog data from IPHaS (Barentsen et al. 2014) and PanSTARRS (Flewelling et al. 2016), Reported $r$-band magnitudes were adjusted (in the case of IPHaS, first from Vega to the AB system) from the PanSTARRS AB system to Gaia's $G$ band, using an

\footnotetext{
12 http://gsaweb.ast.cam.ac.uk/alerts/alert/Gaia17bpi/
} 


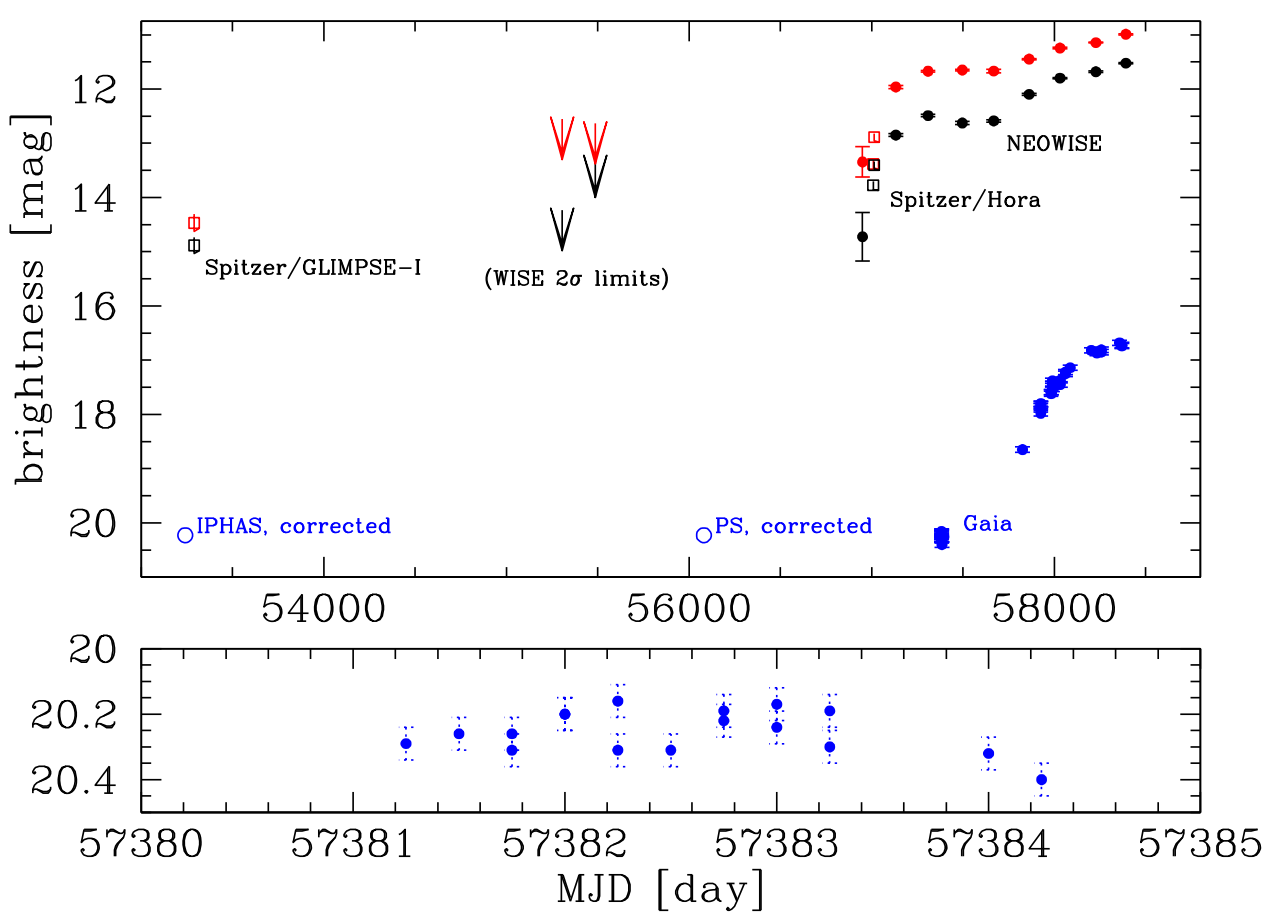

Figure 3. Top: light curves measured by Gaia ( $G$ band with an effective wavelength $0.673 \mu \mathrm{m}$ ) in blue filled symbols and NEOWISE (3.4 and $4.6 \mu \mathrm{m})$ in black and red filled symbols. The recent photometry measuring the outburst is supplemented by previous data (open symbols) from the IPHaS and PanSTARRS surveys in the optical, corrected from $r$ band to $G$ band as described in the text, and from Spitzer in the infrared, plotted as the native 3.6 and $4.5 \mu$ m measurements, without correction. Error bars are shown on all points. Downward pointing arrows indicate the epochs of position coverage by the WISE sky survey, in which the source was not detected. Bottom: zoomed-in to the first epochs of Gaia data, taken in the last days of 2015. Notional 5\% uncertainties are plotted as dotted error bars, derived by inflating the Gaia DR2 uncertainty for the number of transits included in the scatter measurement. If the real uncertainties are at this level or smaller, variability at the several tenths of a magnitude level could have been occurring before the major brightening episode.

Table 2

SIMBAD-identified YSOs within 2 arcmin of Gaia 17bpi

\begin{tabular}{lccc}
\hline \hline Name & R.A. (J2000) & Decl. (J2000) & Separation $($ ") \\
\hline 2MASS J19310037+1827424 & $19: 31: 00.38$ & $+18: 27: 42.4$ & 74.8 \\
SSTGLMC G053.8210-00.0303 & $19: 31: 02.28$ & $+18: 29: 14.0$ & 94.3 \\
SSTGLMC G053.8129-00.0785 & $19: 31: 12.00$ & $+18: 27: 25.0$ & 95.2 \\
2MASS J19310700+1829328 & $19: 31: 07.01$ & $+18: 29: 32.8$ & 102 \\
2MASS J19305884+1829007 & $19: 30: 58.84$ & $+18: 29: 00.7$ & 117 \\
\hline
\end{tabular}

empirical calibration. ${ }^{13}$ Looking to other sources of recent photometry, we discovered that although there is $\mathrm{PTF} / \mathrm{iPTF}$ imaging in the region spanning the time period after the PanSTARRS data point, and before/after the first Gaia detections, the source sits in a PTF chip gap. There is thus unfortunately no optical photometry recording the initial rise of Gaia $17 \mathrm{bpi}$.

After the 2017 Gaia alert was issued, we monitored the object using the Liverpool Telescope (Steele et al. 2004). Three nights of griz photometry were obtained on 2017 June 28, 2017 October 2, and 2018 August 22. A single epoch of $H$-band photometry was obtained on 2017 June 28. The griz magnitudes were calibrated using PanSTARRS AB magnitudes for a set of stars around 5 arcmin from Gaia $17 \mathrm{bpi}$. The $H$-band data were calibrated in a similar way using 2MASS magnitudes. At the time of the first night of monitoring, the object was already 2 mag brighter in both the optical and the infrared than the quiescent

\footnotetext{
13 We derived $G_{\text {calculated, Vega }}=-2.375+1.401 \times r_{\mathrm{PS}, \mathrm{AB}}-0.01694 \times r_{\mathrm{PS}, \mathrm{AB}}^{2}$ over the magnitude range $13<G<22$.
}

state as measured in earlier PanSTARRS and 2MASS data. The measurements are given in Table 3.

Beginning in spring of 2018, as Gaia 17 bpi began to show signs of approaching peak brightness, the Zwicky Transient Facility (ZTF) became operational. Complementing the Gaia light curve, there are several tens of epochs of $g$-band and $r$-band photometry from ZTF taken between 2018 March through November (when the source set for the season). These data will become available with the release of the NSF/MSIP public survey part of ZTF. However, there is a single ZTF measurement from a "reference image" with $g=18.505$ and $r=16.970 \mathrm{mag}$ reported at IPAC/IRSA for a date that cannot be determined at present.

Additional optical imaging was performed with the University of Kent's Beacon Observatory (Froebrich et al. 2018). The telescope is a 17 inch Planewave Corrected Dall-Kirkham Astrograph with a $4 \mathrm{k} \times 4 \mathrm{k}$ Peltier-cooled CCD camera and a $B, V, R, I, \mathrm{H} \alpha$ filter set. The final images for each night in each of the broadband filters were combined from separate integrations. 


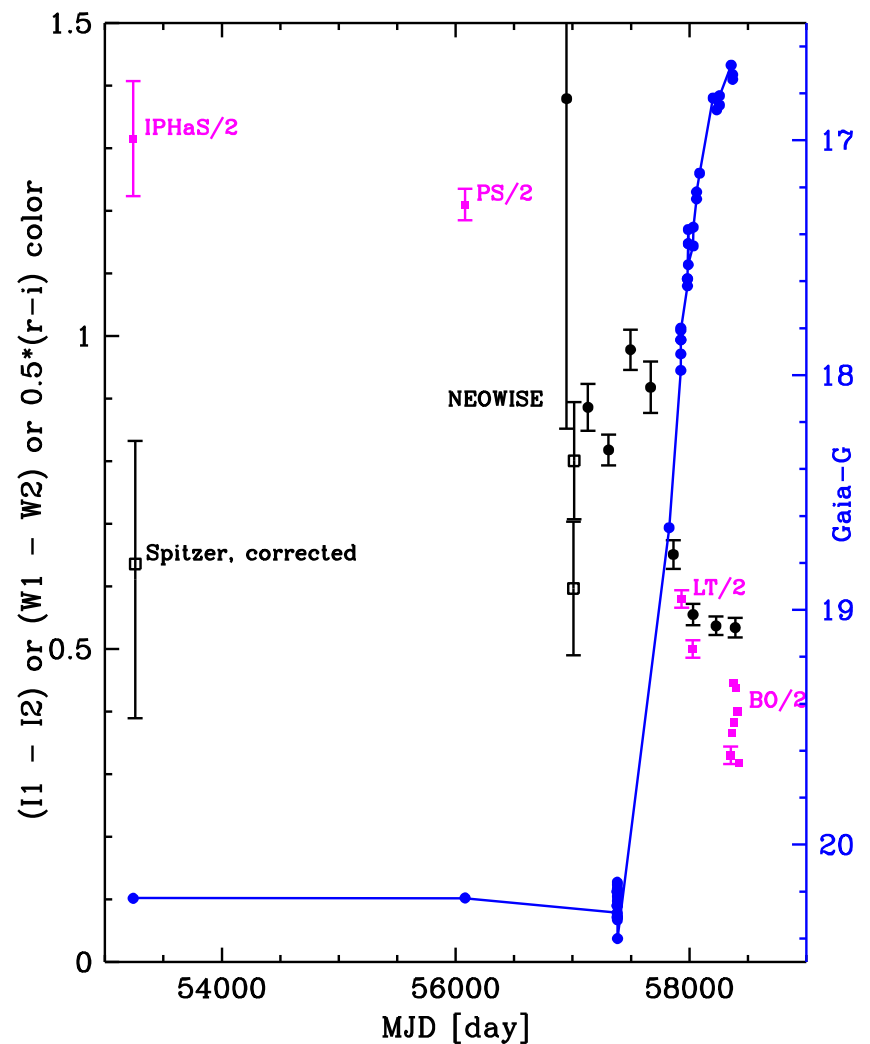

Figure 4. Magenta points illustrate the optical color evolution of Gaia 17bpi, as measured by the post-outburst photometry presented here compared to the pre-outburst IPHaS and PanSTARRS optical color (corrected as necessary from AB to Vega magnitudes). The $r-i$ color becomes bluer during the outburst. The optical color change is substantially larger than indicated by the axis labeling, as the magenta data points have been divided by 2 for purposes of easy comparison to the infrared color, shown in black. Black points illustrate the infrared color evolution of Gaia $17 \mathrm{bpi}$, as measured by NEOWISE and Spitzer, the latter photometry color-corrected to the NEOWISE photometric system; see the text. The [3.4]-[4.6] color in outburst may be initially slightly redder than the pre-outburst color. As Gaia $17 \mathrm{bpi}$ becomes optically bright (blue points, same as in Figure 3), the [3.4]-[4.6] color clearly turns bluer, in concert with the optical color.

Standard data reduction using bias, darks, and sky-flats have been applied. BVri photometric measurements are given in Table 3.

\subsubsection{Infrared}

The position of Gaia 17bpi was observed by WISE (Wright et al. 2010) during its primary mission in 2010 April and October, and again twice per year between 2014 October and 2018 October during the reactivated NEOWISE survey (Mainzer et al. 2014). NEOWISE did not observe the source in 2014 April because it lies in the region of sky that was missed due to a short safehold during that time (Cutri et al. 2015).

Gaia 17bpi was not detected in the WISE observations, and thus it has no entry in the AllWISE Source Catalog. Brightness upper limits for the 2010 April and October observations were estimated using an 8 arcsec aperture on coadds of the 15 individual exposures covering the object in each epoch. The magnitude upper limits were computed by adding two times the flux uncertainty to the measured flux in the aperture.

The source is, however, well detected at 3.4 and $4.6 \mu \mathrm{m}$ in the NEOWISE observations beginning in 2015 April. The midinfrared brightening is illustrated in Figure 5.

Because the field is complicated in the mid-infrared, due to both nebulosity and to very red nearby bright sources, the
NEOWISE photometry was validated by examining individual images. Profile-fit photometric measurements from each individual exposure are available in the NEOWISE Source Database $^{14}$ (Cutri et al. 2015). For the NEOWISE 2015 April and later epochs, the magnitudes and uncertainties listed in Table 4 were computed from the mean and standard deviation of the mean of the Source Database profile-fit fluxes from all exposures taken during each epoch.

For the 2014 October observation set, the NEOWISE Source Database has only one reported detection with an unusually large positional offset relative to the later epochs. The source is not obvious in the individual 2014 October exposures, and we deemed the one measurement to be spurious. However, the source is faintly visible at the correct position in a coadded image formed by combining the 15 individual exposures covering the position, as shown in Figure 5. Because of the complexity of the surrounding field, 3.4 and $4.6 \mu \mathrm{m}$ photometry was performed on difference images that were constructed by subtracting coadded 2010 October images (where the source is not detected) from the coadded 2014 October images to suppress the nearby confusing objects. The flux of the source was measured in an 8 arcsec radius aperture on the difference images, which is the same sized aperture used for the "standard aperture" photometry in NEOWISE automated data processing. The aperture measurements on the difference images were put on the photometric scale of the automated profile-fit measurements from the later epochs by applying the same aperture corrections and normalization used in the automated processing (these are the w1mcor and w2mcor parameters in the NEOWISE Source Database entries). This procedure was verified by generating difference images for the later NEOWISE epochs when the source was well detected. The calibrated aperture measurements on the difference images agree with the average profile-fit measurements to within $11 \%$ in $W 1$ and 6\% in W2 in each epoch (excluding 2014 October when the source was too faint to be reliably detected in the individual exposures). The results appear in Table 4.

The assembled light curve includes the Spitzer/GLIMPSE-I photometry from 2004 discussed above. In addition, there is a second set of Spitzer data, taken in 2014 December, just after the first NEOWISE measurements from 2014 October. The nearby infrared dark cloud was targeted twice with IRAC 3.6 and $4.5 \mu \mathrm{m}$ imaging, observed as part of program 10012 (PI: J. Hora). Each of these observations are much deeper than GLIMPSE, with integration time $\sim 150 \mathrm{~s}$ as compared to $\sim 4 \mathrm{~s}$, and the source was brighter by this time as well. Aperture photometry was performed as described earlier for the GLIMPSE re-reduction, but using aperture corrections of 1.125 and 1.120, as appropriate for postcryogenic Spitzer data. The results appear in Table 4.

The two epochs of 2014 Spitzer data appear to have captured the outburst in progress, with the second epoch brighter than the first (by $0.4 \mathrm{mag}$ at $3.6 \mu \mathrm{m}$ and $0.5 \mathrm{mag}$ at $4.5 \mu \mathrm{m}$ ), which we confirmed from visual inspection and repeated photometric measurement. The only slightly earlier 2014 NEOWISE measurement is fainter, and the first 2015 NEOWISE measurement is brighter (see Figure 3).

\footnotetext{
${ }^{14}$ NEOWISE; http://wise2.ipac.caltech.edu/docs/release/neowise/expsup.
} 


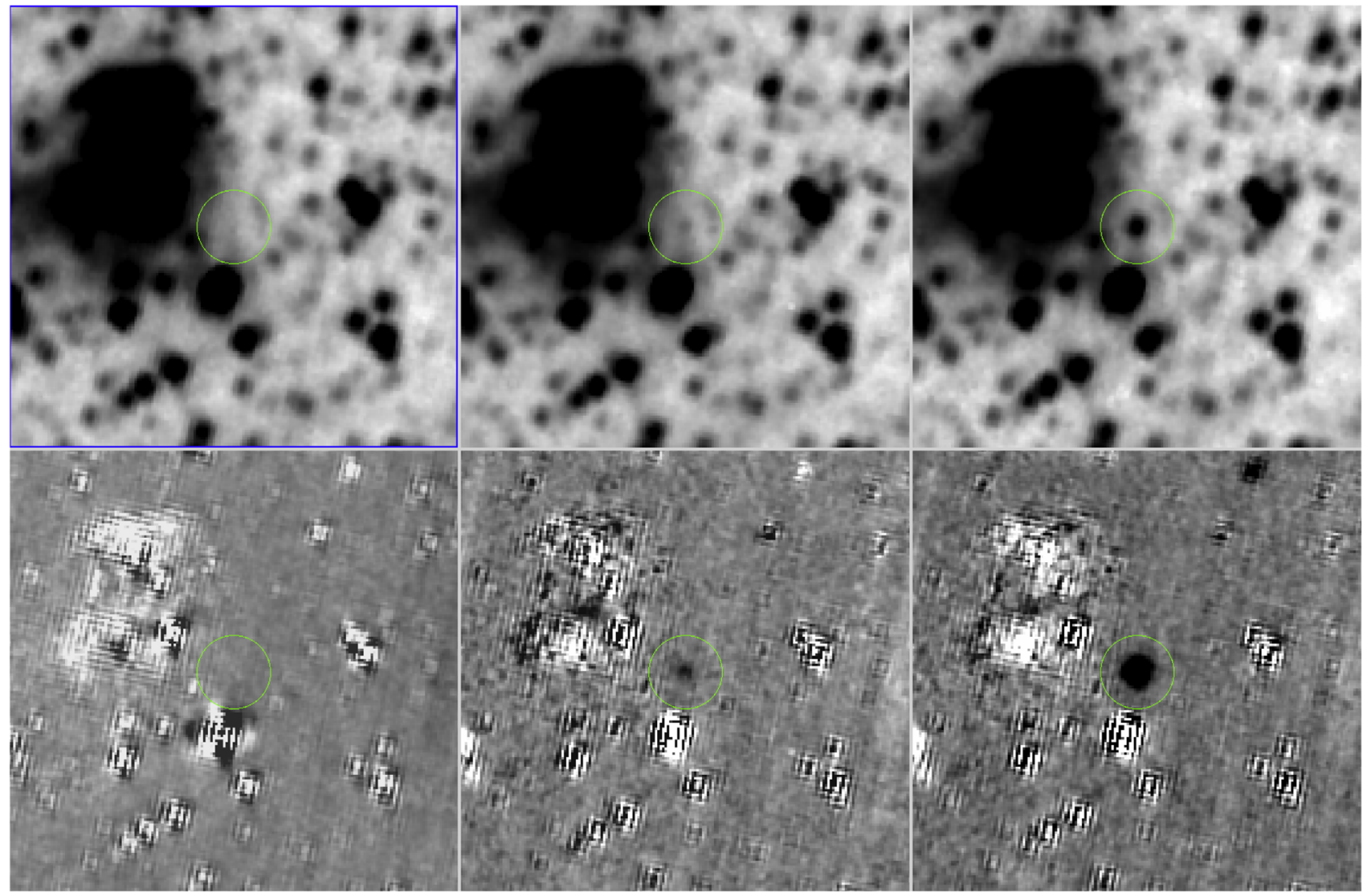

Figure 5. Top row shows the NEOWISE direct coadded $4.6 \mu \mathrm{m}$ images from (left to right) 2010 October, 2014 October, and 2017 October. Bottom row shows the difference images relative to an earlier epoch, specifically, (left to right) between 2010 October and 2010 April, between 2014 October and 2010 October, and between 2017 October and 2010 October. Fields of view are $3^{\prime} \times 3^{\prime}$; green circles are $30^{\prime \prime}$ in diameter, centered on the position of the source. North is toward the top, and east is toward the left.

\subsection{Quantitative Analysis of the Light Curves and Color Curves}

Figure 3 shows the assembled optical and infrared lightcurve data. Unfortunately, the beginning of the turn-up from quiescence to an early outburst stage is not well observed in either the optical or the infrared. However, the later parts of the optical rise and the optical peak are adequately sampled, even at the low cadence of Gaia. As of 2018 September, Gaia 17bpi is $\sim 3.5 \mathrm{mag}$ brighter in the optical and $\sim 3$ mag brighter in the mid-infrared than it was 5-15 yr ago.

The first Gaia measurements in late 2015 indicate no change in brightness from the historical optical photometry. However, about a year before, in late 2014, there is a pair of Spitzer measurements that are already $1.1 \mathrm{mag}$ brighter in both $I 1$ and $I 2$ than the Spitzer I1 and $I 2$ photometry from $10 \mathrm{yr}$ earlier, in 2004. The first NEOWISE points were taken just before the two Spitzer measurements and $1.2 \mathrm{yr}$ earlier than the first Gaia measurement. They have large errors, and while the $3.4 \mu \mathrm{m}$ point is not significantly brighter than the earlier Spitzer measurement, the $4.6 \mu \mathrm{m}$ measurement could be. The outburst may have begun between October and December of 2014.

The evidence from the time series suggests that the outburst may have started in the infrared and manifested later in the optical. As we do not sample the beginning of the burst in the optical, given the large time gap between the initial and subsequent Gaia photometry, we cannot calculate the time delay, but $\sim 1.5-2 \mathrm{yr}$ is implied. The NEOWISE light curve further indicates a possible two-stage rise, in which there is perhaps 18 months of measurable mid-infrared brightening, followed by a plateau of about a year, then another at least 24 months of midinfrared brightening corresponding to the optical rise that is well sampled by the Gaia measurements. Figure 6 shows our analytic fit to the optical and mid-infrared light curves.

We fit the existing pre-outburst and outburst optical photometry with a sigmoid function, assuming the peak brightness occurs at the last measured epoch, which is defined as $t=0$. The sigmoid is described by $L /\left(1+e^{\left(-k \times\left(t-t_{o}\right) / \text { days }\right)}\right)$ and our best-fit parameter values are $t_{o}=-522.9 \pm 0.9$ (meaning that the outburst starts $2 \times t_{o}=1046$ days before its peak), $k=-0.00728 \pm 0.00043$ (where the $1 / k$ value represents a timescale for the rise, in this case a 137.4 day e-folding), and $L=3.541 \pm 0.002$ (representing the full amplitude of the rise). We note that it is not yet clear whether Gaia 17bpi has reached its peak brightness. If it continues to rise, all of the numbers quoted above would increase.

Applying the same methodology to the infrared data, we fit the first and second plateaus in the NEOWISE light curve separately. We find that the first rise has a 71 day e-folding time, taking $2 \times t_{o}=593$ days to rise $2.2 \mathrm{mag}$, while the second rise has a 149 day e-folding time, taking $2 \times t_{o}=998$ days to rise another $1.1 \mathrm{mag}$. The second rise in the NEOWISE data appears to correspond to the brightening in the Gaia data. Again, it is not clear whether the second infrared rise has reached its peak, so the numbers above could be lower limits. 
Table 3

Optical Photometric Follow-up during the Outburst of Gaia 17bpi

\begin{tabular}{|c|c|c|c|c|c|}
\hline Telescope & MJD & Magnitude & Error & Filter & Photometric System \\
\hline Liverpool & 57933.091466 & 20.12 & 0.02 & $g$ & PanSTARRS (AB) \\
\hline Liverpool & 57933.097860 & 18.47 & 0.01 & $r$ & PanSTARRS (AB) \\
\hline Liverpool & 57933.100280 & 17.52 & 0.01 & $i$ & PanSTARRS (AB) \\
\hline Liverpool & 58028.982488 & 18.97 & 0.02 & $g$ & PanSTARRS (AB) \\
\hline Liverpool & 58028.986326 & 17.55 & 0.01 & $r$ & PanSTARRS (AB) \\
\hline Liverpool & 58028.987833 & 16.76 & 0.01 & $i$ & PanSTARRS (AB) \\
\hline Liverpool & 58353.054681 & 16.91 & 0.01 & $r$ & PanSTARRS (AB) \\
\hline Liverpool & 58353.056184 & 16.22 & 0.01 & $i$ & PanSTARRS (AB) \\
\hline Liverpool & 58353.056767 & 15.77 & 0.01 & $z$ & PanSTARRS (AB) \\
\hline Liverpool & 57933.104537 & 13.48 & 0.02 & $H$ & 2MASS \\
\hline Beacon & 58362.43 & 17.474 & 0.067 & V & APASS \\
\hline Beacon & 58379.36 & 16.051 & 0.108 & $i$ & APASS \\
\hline Beacon & 58383.35 & 17.575 & 0.060 & V & APASS \\
\hline Beacon & 58383.35 & 16.783 & 0.083 & $r$ & APASS \\
\hline Beacon & 58383.36 & 16.027 & 0.099 & $i$ & APASS \\
\hline Beacon & 58395.37 & 19.254 & 0.135 & $B$ & APASS \\
\hline Beacon & 58395.36 & 17.667 & 0.062 & $V$ & APASS \\
\hline Beacon & 58395.36 & 17.024 & 0.084 & $r$ & APASS \\
\hline Beacon & 58395.36 & 16.149 & 0.096 & $i$ & APASS \\
\hline Beacon & 58412.31 & 17.651 & 0.070 & V & APASS \\
\hline Beacon & 58412.31 & 16.902 & 0.078 & $r$ & APASS \\
\hline Beacon & 58412.31 & 16.101 & 0.097 & $i$ & APASS \\
\hline Beacon & 58425.29 & 17.420 & 0.063 & V & APASS \\
\hline
\end{tabular}

Table 4

Newly Measured Infrared Photometry before and during the Outburst of Gaia 17bpi

\begin{tabular}{|c|c|c|c|c|c|}
\hline Telescope & MJD & $3.4 \mu \mathrm{m}$ Magnitude & Error & $4.6 \mu \mathrm{m}$ Magnitude & Error \\
\hline WISE & 55305.20 & $>14.23$ & $2 \sigma$ limit & $>12.55$ & $2 \sigma$ limit \\
\hline WISE & 55487.10 & $>13.25$ & $2 \sigma$ limit & $>12.63$ & $2 \sigma$ limit \\
\hline NEOWISE & 56949.30 & 14.72 & 0.45 & 13.35 & 0.28 \\
\hline Spitzer & 57008.16 & $13.78(3.6 \mu \mathrm{m})$ & 0.08 & $13.38(4.5 \mu \mathrm{m})$ & 0.08 \\
\hline Spitzer & 57014.19 & $13.40(3.6 \mu \mathrm{m})$ & 0.07 & $12.89(4.5 \mu \mathrm{m})$ & 0.07 \\
\hline NEOWISE & 57131.80 & 12.85 & 0.03 & 11.96 & 0.03 \\
\hline NEOWISE & 57308.40 & 12.49 & 0.02 & 11.67 & 0.01 \\
\hline NEOWISE & 57495.90 & 12.63 & 0.03 & 11.65 & 0.01 \\
\hline NEOWISE & 57667.70 & 12.59 & 0.02 & 11.67 & 0.03 \\
\hline NEOWISE & 57861.90 & 12.10 & 0.02 & 11.45 & 0.01 \\
\hline NEOWISE & 58030.80 & 11.80 & 0.01 & 11.24 & 0.01 \\
\hline NEOWISE & 58227.20 & 11.68 & 0.01 & 11.15 & 0.01 \\
\hline NEOWISE & 58391.40 & 11.52 & 0.01 & 10.99 & 0.01 \\
\hline
\end{tabular}

The amplitude of the photometric brightening corresponds to a luminosity increase by a factor of 25 , from the estimated $0.3 L_{\odot}$ for the progenitor T Tauri star, to $7.5 L_{\odot}$ in outburst.

Figure 4 illustrates the optical and mid-infrared color changes that have been observed. Although there is little optical color data available for Gaia 17bpi in the outburst phase (Table 3), compared to pre-outburst colors from IPHaS and PanSTARRS, the source is clearly bluer. After accounting for $\mathrm{AB}$ to Vega magnitude transformations, the blueing is about $1.2 \mathrm{mag}$ in $r-i$ color and $0.8 \mathrm{mag}$ in $g-r$ color. There should be a full $g-r$ color time series available from ZTF when the first data release occurs. In addition, a future data release from Gaia should contain $B P$ and $R P$ measurements at the same epochs as the $G$ measurements.

In the infrared, the Spitzer measurements show no infrared color change between the 2004 and 2014 data. However, the 


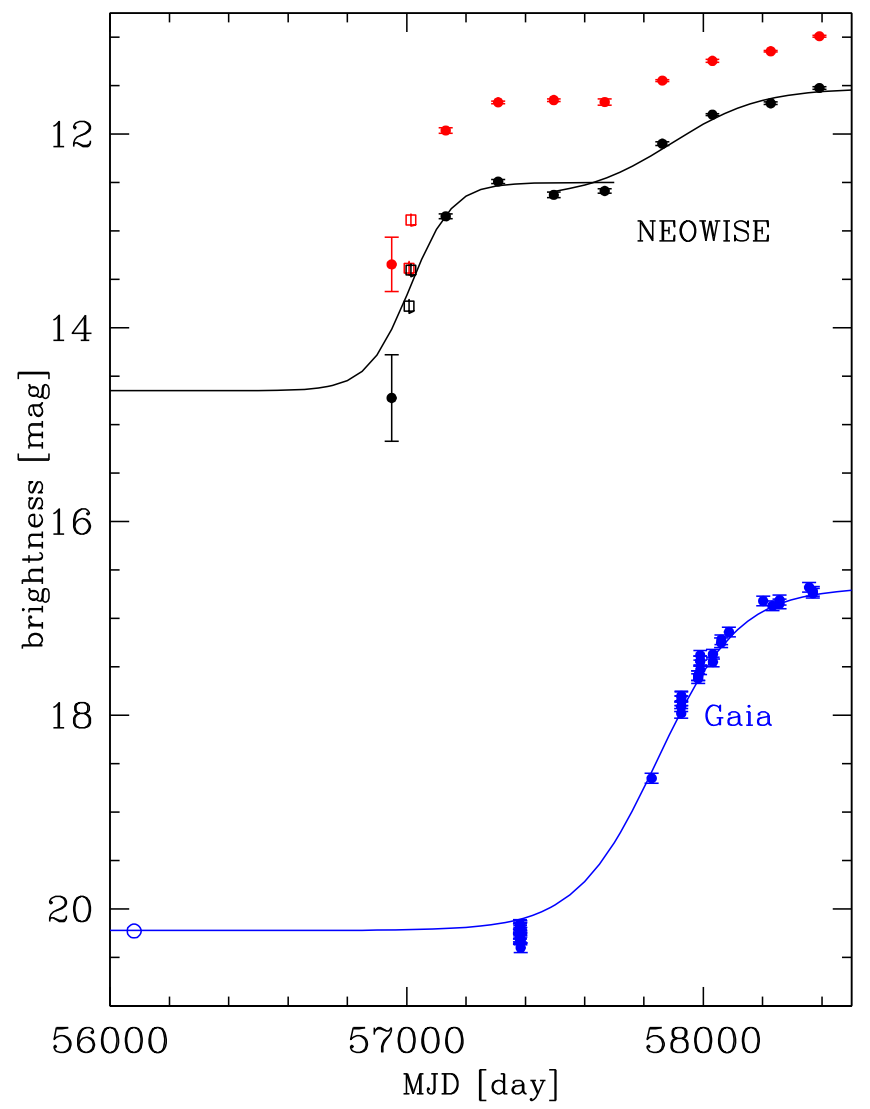

Figure 6. Analytic fits to the FU Ori-type outburst of Gaia 17bpi. The Gaia optical light curve is fit with a single sigmoid function, while the NEOWISE mid-infrared light curve is modeled as having two stages, described by two sigmoids. See the text for fit parameters.

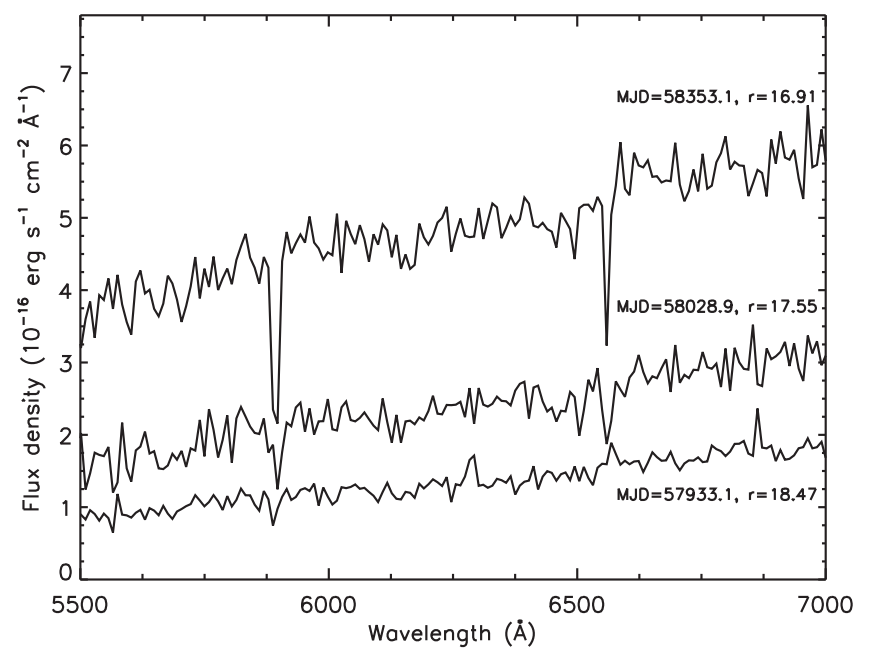

Figure 7. Portion of the Liverpool Telescope spectra of Gaia 17bpi at $R=350$. From bottom to top, representing three different nights during the light-curve rise, $\mathrm{NaD}$ and $\mathrm{H} \alpha$ absorption appears and strengthens. The data used to create this figure are available.

first NEOWISE measurement seems to indicate a redder [3.4][4.6] color (a.k.a. $W 1-W 2$ ) compared to the historical Spitzer [3.6]-[4.5] color (a.k.a. $I 1-I 2$ ), by $\sim 0.45$ mag. Color terms between the Spitzer and WISE filter systems cannot explain the amplitude of the apparent color change; green points in
Figure 4 show the Spitzer photometry corrected to the NEOWISE system using an empirically derived relation. ${ }^{15}$ The NEOWISE outburst photometry is initially redder, but as the optical and second-stage-infrared burst proceeds, the midinfrared color is observed to become bluer, by $\sim 0.4 \mathrm{mag}$. The fitted color-magnitude slope is $W 1=2.07 \times(W 1-W 2)$ $+10.70 \mathrm{mag}$ with $\mathrm{rms}=0.13 \mathrm{mag}$. This slope is inconsistent with the clearing of extinction.

The evidence for significant and substantial blueing during the outburst, in both optical and infrared colors, points to a dramatic heating event in Gaia $17 \mathrm{bpi}$.

\subsection{Spectroscopic Follow-up Data and Analysis}

During the light-curve rise, the Liverpool Telescope was used to obtain optical spectra using the low-resolution spectrograph SPRAT $(R=350)$. The observations were carried out during the same nights as the photometric monitoring reported above. The data are displayed in Figure 7.

The first low-resolution spectrum, obtained when the source was $\sim 2$ mag brighter than quiescence, shows little in terms of emission or absorption. But as the source brightens to more than $\sim 3$ mag above quiescence, distinct absorption features in $\mathrm{NaD}$ and $\mathrm{H} \alpha$ appear and strengthen over time.

The Keck/DEIMOS (Faber et al. 2003) spectrograph was then used on 2018 September 10 UT by E. N. Kirby to observe Gaia $17 \mathrm{bpi}$ in a $900 \mathrm{~s}$ integration. The $600 \mathrm{ZD}$ grating provided dispersion of $0.65 \AA$ pix $^{-1}$ and led to a resolution of $3.3 \AA$ per 0 " 7 slit, or $R \approx 2200$ over $4630-9865 \AA$, with a small gap from 7211 to $7229 \AA$.

The DEIMOS spectrum (Figure 8) exhibits numerous strong P-Cygni-type blueshifted absorption lines, with accompanying weak redshifted emission components. These profiles are seen in $\mathrm{NaD}, \mathrm{H} \alpha$, and the $\mathrm{Ca}$ II triplet as highlighted in Figure 9. Blueshifted absorption, but non-P-Cygni profiles are presented by the Mgb triplet, K I, and O I. All of the above lines indicate a strong wind with a velocity of several hundred $\mathrm{km} \mathrm{s}^{-1}$. The terminal velocity of the $\mathrm{H} \alpha$ profile, for example, is approximately $-500 \mathrm{~km} \mathrm{~s}^{-1}$. Li I absorption is also present (Figure 9) and has some evidence for a wind component as well. The Li I equivalent width $W_{\lambda}$ is $0.47 \AA$ and is notable relative to the nearby Ca I $6717 \AA$ line with $W_{\lambda}=0.39 \AA$, though there could be minor contamination from weak Fe I lines at 6703.6 and $6705.1 \AA$.

The $\mathrm{H} \alpha$ profile has $W_{\lambda}=9.2 \AA$ in blueshifted absorption with a double-trough, and then a redshifted emission component with $W_{\lambda}=-1.1 \AA$. In $\mathrm{H} \beta$ there is $W_{\lambda}=5.1 \AA$ strength in blueshifted absorption and no emission component. The Ca II $8542 \AA$ line has $W_{\lambda}=5.2 \AA$ in its blueshifted absorption and $W_{\lambda}=-1.3 \AA$ in redshifted emission.

In addition to the lines indicating activity and youth, the optical spectrum of Gaia 17bpi also has numerous neutral species absorption lines that are typical of GK-type spectra, e.g., Fe I, Mg I, Ca I. Also like many FU Ori stars, Gaia 17bpi has a strong feature at $6497 \AA$ that is associated with a Ba II/ $\mathrm{Ca} \mathrm{I} / \mathrm{Fe}$ I blend.

The Palomar 200" telescope and TripleSpec (Herter et al. 2008) instrument were used on 2018 September 25 UT to observe Gaia 17bpi in the YJHK bands. A total of $1 \mathrm{hr}$ of integration was accumulated from three sets of four (A-B-B-A)

$\overline{15}(W 1-W 2)=1.62 \times(I 1-I 2)-0.04 \mathrm{mag}$, with rms $=0.24 \mathrm{mag}$. 


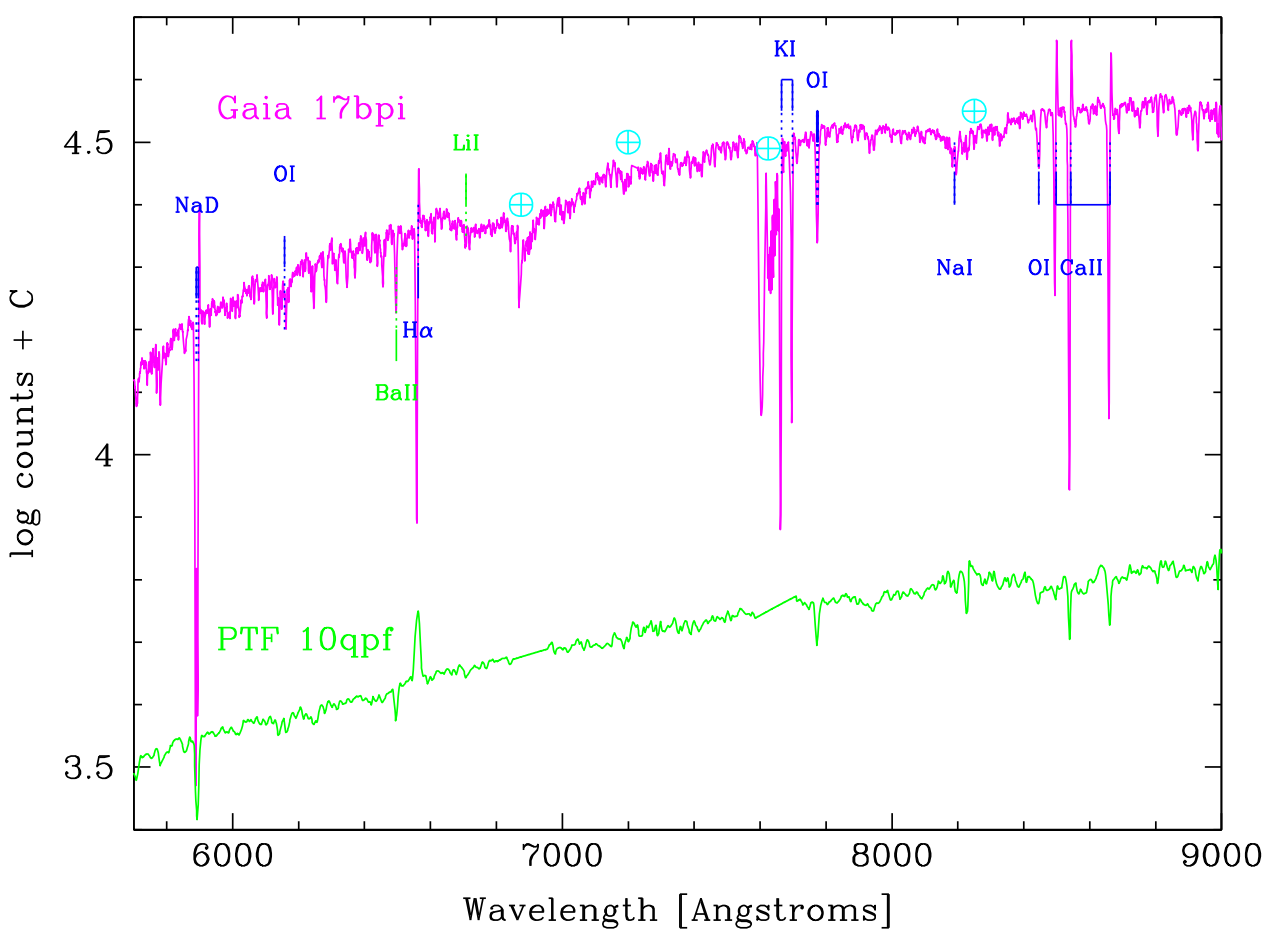

Figure 8. Portion of the Keck/DEIMOS spectrum of Gaia 17bpi at $R=2200$. Prominent spectral lines are labeled, with the $\oplus$ symbol indicating regions of significant telluric contamination. The spectrum is consistent with that of a GK-type photosphere and is comparable to the spectrum of PTF 10qpf = V2493 Cyg (formerly known as $\mathrm{LkH} \alpha 188 / \mathrm{G} 4=\mathrm{HBC}$ 722), which was observed at a similar time in its outburst, though at lower resolution and with the telluric-contaminated regions interpolated over. The data used to create this figure are available.

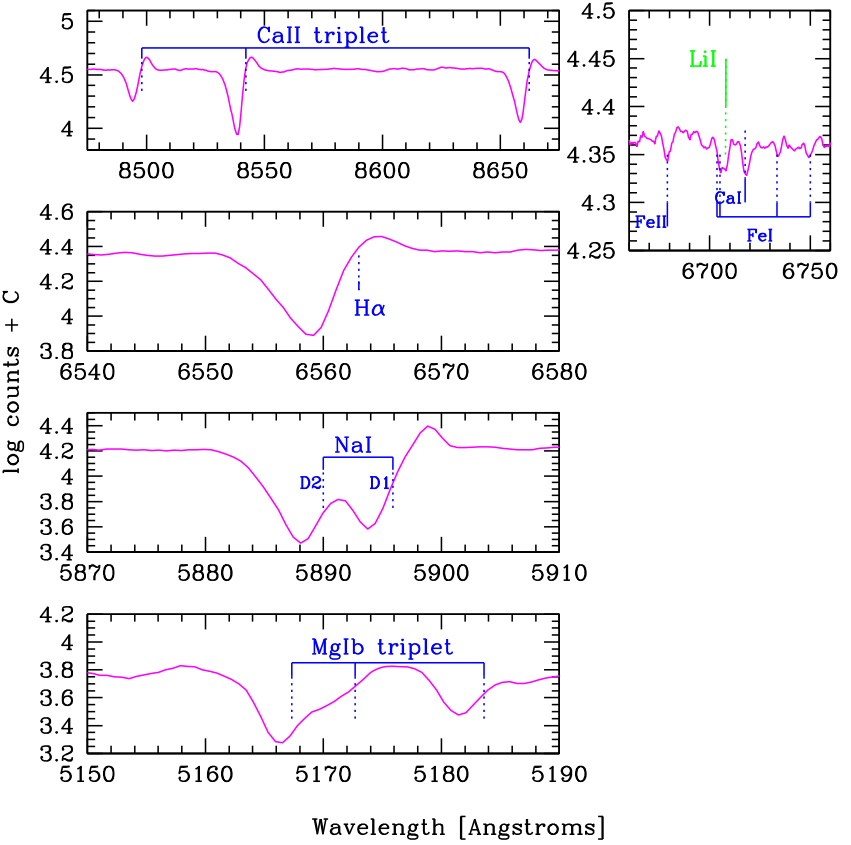

Figure 9. Zoomed-in on portions of Figure 8 illustrating the P-Cygni nature of the $\mathrm{NaD}, \mathrm{H} \alpha$, and $\mathrm{Ca}$ II line profiles, as well as the blueshifted absorption in the $\mathrm{Mgb}$ triplet lines-all signatures of a strong wind. Gaia $17 \mathrm{bpi}$ clearly exhibits Li I absorption, which appears to also have a blueshifted component that exceeds possible contributions from weak Fe I lines.

nods. Data were reduced using a version of the XSpexTool developed by $M$. Cushing and W. Vacca adapted for TripleSpec at Palomar. The spectrum has resolution $R=2700$ and is illustrated in Figure 10.
The classic FU Ori signatures of ${ }^{12} \mathrm{CO}$ absorption in the $2.3 \mu \mathrm{m}$ region and prominent $\mathrm{H}_{2} \mathrm{O}$ absorption in the $K$ band and $H$ band are exhibited, as are absorption features due to various atomic lines. Notably, He I 10,830 ̊ and several H I Pa lines are apparent. Although not apparent at our resolution, these lines likely have P-Cyg-type wind profiles similar to those exhibited by the optical region and $\mathrm{HI}$ Balmer and Ca II lines. We refer to the FU Ori spectral atlas of Connelley \& Reipurth (2018), and specifically to their Figure 3, for a set of comparison objects.

\section{Discussion and Conclusions}

There is wide diversity among members of the FU Ori class regarding outburst rise times and amplitudes. Gaia 17bpi has exhibited a $3.5 \mathrm{mag}$ rise in the optical over 1046 days, with a 137.4 day e-folding time and an accompanying $>3 \mathrm{mag}$ brightness increase in the mid-infrared. The infrared brightening took place in two stages, the first beginning approximately $1.5 \mathrm{yr}$ before the optical brightening (late in 2014 and into 2015, compared to in 2017), and the second seemingly coincident with the optical brightening. Source colors show both mid-infrared and optical blueing during the lightcurve rise.

The unifying elements of the FU Ori class are the distinctive spectroscopic features, notably a spectral change from hotter to cooler with increasing wavelength through the optical and nearinfrared, as well as the appearance of strong wind signatures that are a consequence of the onset of rapid accretion. Gaia 17bpi exhibits a GK-type absorption spectrum in the optical and an M-type absorption spectrum in the infrared, as is typical of the FU Ori class. While the spectra are similar in the different wavelength ranges to these single-temperature 


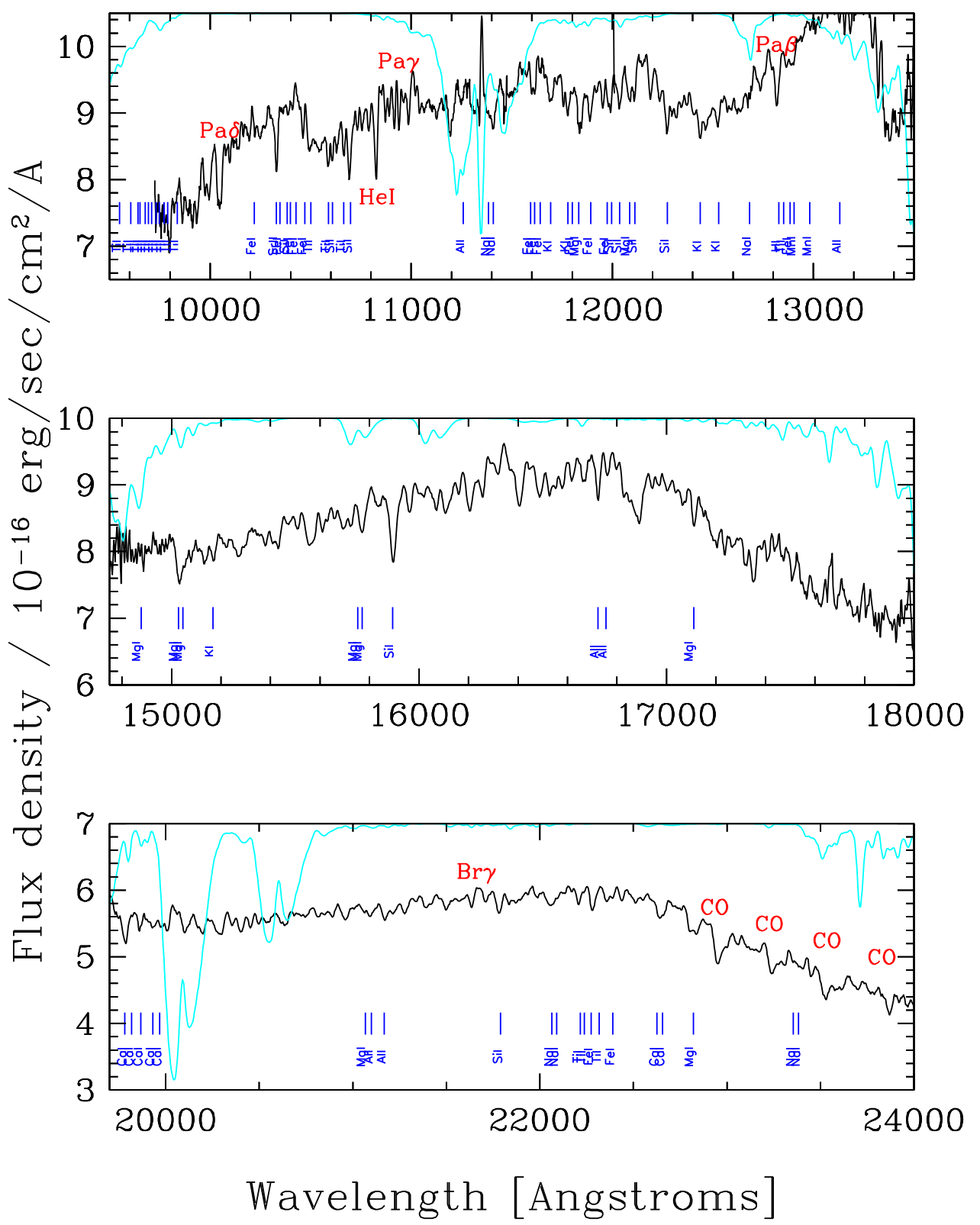

Figure 10. Portion of the Palomar/TripleSpec spectrum of Gaia 17bpi at $R=2700$. Prominent spectral lines are labeled. The cyan line is a model atmospheric transmission spectrum plotted on a vertical scale from $0 \%$ to $100 \%$ and indicates regions where the telluric correction applied to the data is large and possibly uncertain. The Gaia 17bpi spectrum is similar to that of an M-type photosphere, mainly due to the prominent $\mathrm{H}_{2} \mathrm{O}$ broad depressions in the $H$ band and $K$ band and the ${ }^{12} \mathrm{CO}(2-0)$ bandhead absorption in the $K$ band. The spectrum agrees well with the set of FU Ori objects displayed in Figure 3 of Connelley \& Reipurth (2018). The data used to create this figure are available.

spectral classes, they are not exact matches given the complexity of the "photospheres" of members of the FU Ori class. Gaia 17bpi also shows spectroscopic signatures of strong wind/outflow, and it displays the Li I $6707 \AA$ signature of youth. These characteristics are also consistent with an FU Ori classification.

With the data in hand to date, Gaia 17bpi appears to meet the photometric and spectroscopic criteria of a bona fide FU Ori source. The source thus joins V900 Mon (Thommes et al. 2011; Reipurth et al. 2012), V960 Mon (Maehara et al. 2014; Hillenbrand 2014), [CTF93]216-2 = V2775 Ori (Caratti o Garatti et al. 2011), and HBC 722 = V2493 Cyg (Semkov et al. 2010; Miller et al. 2011) as the latest entries over the past 10 years in this still-rare category. Fewer than 13 of the known $\sim 25$ FU Ori objects have been observed to undergo their dramatic brightness increases, with the rest classified as such only after the fact, e.g., V582 Aur (Munari et al. 2009; Samus 2009) was relatively recently recognized as a member of the class, in 2009, but likely outburst in the mid1980s (Semkov et al. 2013).

Gaia 17bpi is unique among FU Ori outbursts in having its photometric brightening detected at both optical and midinfrared wavelengths. The burst appears to have started in the infrared, consistent with disk models that predict instabilities in the inner $0.5-1$ au of protostellar and $\mathrm{T}$ Tauri accretion disks as the origin of FU Ori events. 
We acknowledge ESA Gaia, DPAC, and the Photometric Science Alerts Team, as well as NASA's NEOWISE team. We are extremely grateful to Evan Kirby for obtaining and reducing to 1D format the Keck/DEIMOS spectrum presented here. We are also grateful to the Palomar Observatory staff for their real-time assistance in obtaining the TripleSpec spectrum presented here. Conversations with Kevin Burdge and Sean Carey about various photometric survey data sets were beneficial in our work. The contributions of C.C.P. and T.N. were funded by a Leverhulme Trust Research Project Grant and of S.M. through a Science and Technology Facilities Council studentship.

Facilities: Gaia, LT:IO:O, LT:IO:I, BO, Hale:DBSP, Hale: TSPEC, Keck:I:DEIMOS, IPHaS, PanSTARRS, 2MASS, Spitzer, Herschel, WISE, NEOWISE, IRSA.

\section{ORCID iDs}

Sam Morrell (1) https://orcid.org/0000-0001-6352-5312 Tim Naylor (i) https://orcid.org/0000-0002-0506-8501 Michael A. Kuhn (1) https://orcid.org/0000-0002-0631-7514 Roc M. Cutri (1) https://orcid.org/0000-0002-0077-2305 Luisa M. Rebull (1) https://orcid.org/0000-0001-6381-515X

\section{References}

Anderson, L. D., Bania, T. M., Balser, D. S., et al. 2014, ApJS, 212, 1 Bae, J., Hartmann, L., Zhu, Z., \& Nelson, R. P. 2014, ApJ, 795, 61 Barentsen, G., Farnhill, H. J., Drew, J. E., et al. 2014, MNRAS, 444, 3230 Barentsen, G., Vink, J. S., Drew, J. E., \& Sale, S. E. 2013, MNRAS, 429, 1981 Benjamin, R. A., Churchwell, E., Babler, B. L., et al. 2003, PASP, 115, 953 Caratti o Garatti, A., Garcia Lopez, R., Scholz, A., et al. 2011, A\&A, 526, L1 Carey, S. J., Noriega-Crespo, A., Mizuno, D. R., et al. 2009, PASP, 121, 76 Churchwell, E., Babler, B. L., Meade, M. R., et al. 2009, PASP, 121, 213

Cody, A. M., \& Hillenbrand, L. A. 2018, AJ, 156, 71

Connelley, M. S., \& Reipurth, B. 2018, ApJ, 861, 145

Contreras Peña, C., Lucas, P. W., Kurtev, R., et al. 2017a, MNRAS, 465, 3039 Contreras Peña, C., Lucas, P. W., Minniti, D., et al. 2017b, MNRAS, 465, 3011

Cutri, R. M., Mainzer, A., Conrow, T., et al. 2015, Explanatory Supplement to the NEOWISE Data Release Products (Washington, DC: NASA)
Cutri, R. M., Skrutskie, M. F., van Dyk, S., et al. 2003, The IRSA 2MASS AllSky Point Source Catalog: NASA/IPAC Infrared Science Archive (Washington, DC: NASA)

Faber, S. M., Phillips, A. C., Kibrick, R. I., et al. 2003, Proc. SPIE, 4841, 1657 Flewelling, H. A., Magnier, E. A., Chambers, K. C., et al. 2016, arXiv:1612.05243 Froebrich, D., Campbell-White, J., Scholz, A., et al. 2018, MNRAS, 478, 5091 Froebrich, D., Davis, C. J., Ioannidis, G., et al. 2011, MNRAS, 413, 480

Gaia Collaboration, Brown, A. G. A., Vallenari, A., et al. 2018, A\&A, 616, A1

Gutermuth, R. A., Megeath, S. T., Myers, P. C., et al. 2009, ApJS, 184, 18

Hartmann, L., Herczeg, G., \& Calvet, N. 2016, ARA\&A, 54, 135

Hartmann, L., \& Kenyon, S. J. 1996, ARA\&A, 34, 207

Hauschildt, P. H., Allard, F., Ferguson, J., Baron, E., \& Alexander, D. R. 1999, ApJ, 525, 871

Herbig, G. H. 1977, ApJ, 217, 693

Herter, T. L., Henderson, C. P., Wilson, J. C., et al. 2008, Proc. SPIE, 7014, $70140 \mathrm{X}$

Hillenbrand, L. 2014, ATel, 6797

Hillenbrand, L. A., \& Findeisen, K. P. 2015, ApJ, 808, 68

Hodgkin, S. T., Wyrzykowski, L., Blagorodnova, N., \& Koposov, S. 2013, RSPTA, 371, 20120239

Kim, H.-J., Koo, B.-C., \& Davis, C. J. 2015, ApJ, 802, 59

Kuhn, M. A., Hillenbrand, L. A., Sills, A., Feigelson, E. D., \& Getman, K. V. 2019, ApJ, in press (arXiv: 1807.02115)

Maehara, H., Kojima, T., \& Fujii, M. 2014, ATel, 6770

Mainzer, A., Bauer, J., Cutri, R. M., et al. 2014, ApJ, 792, 30

Miller, A. A., Hillenbrand, L. A., Covey, K. R., et al. 2011, ApJ, 730, 80

Molinari, S., Swinyard, B., Bally, J., et al. 2010, PASP, 122, 314

Munari, U., Siviero, A., Ochner, P., Fiorucci, M., \& Dallaporta, S. 2009, CBET, 1898,

Pilbratt, G. L., Riedinger, J. R., Passvogel, T., et al. 2010, A\&A, 518, L1

Pineda, J. L., Goldsmith, P. F., Chapman, N., et al. 2010, ApJ, 721, 686

Rebull, L. M., Stauffer, J. R., Cody, A. M., et al. 2018, AJ, 155, 196

Reipurth, B. 2016, Inst. Ast. Spec. Publ., 1

Reipurth, B., \& Aspin, C. 2010, in VAOA Conf. Evolution of Cosmic Objects through their Physical Activity, ed. H. A. Harutyunian, A. M. Mickaelian, \& Y. Terzian (Yerevan: NAS RA), 19

Reipurth, B., Aspin, C., \& Herbig, G. H. 2012, ApJL, 748, L5

Samus, N. 2009, CBET, 1896, 1

Semkov, E. H., Peneva, S. P., Munari, U., Milani, A., \& Valisa, P. 2010, A\&A, 523, LL3

Semkov, E. H., Peneva, S. P., Munari, U., et al. 2013, A\&A, 556, A60

Skrutskie, M. F., Cutri, R. M., Stiening, R., et al. 2006, AJ, 131, 1163

Steele, I. A., Smith, R. J., Rees, P. C., et al. 2004, Proc. SPIE, 5489, 679

Thommes, J., Reipurth, B., Aspin, C., \& Herbig, G. H. 2011, CBET, 2795, 1 Vorobyov, E. I., \& Basu, S. 2015, ApJ, 805, 115

Werner, M. W., Roellig, T. L., Low, F. J., et al. 2004, ApJS, 154, 1

Wright, E. L., Eisenhardt, P. R. M., Mainzer, A. K., et al. 2010, AJ, 140, 1868 